\title{
Validación de la prueba de detección de consumo de alcohol, tabaco y sustancias (ASSIST) en pacientes con trastorno psiquiátrico ingresados en una unidad de agudos
}

\section{Validation of the Alcohol Smoking and Substance Involvement Screening Test (ASSIST) in acute psychiatric inpatients}

\author{
Ana Isabel López-Lazcano*, Hugo López-Pelayo*, Mercè Balcells-Oliveró*, Lidia Segura**, \\ Antoni Gual Solé*. \\ * Grup Recerca Addicions Clínic (GRAC-GRE). Departamento de Psiquiatría, Instituto Clínic de Neurociencias. Hospital Clínic i \\ Universitari de Barcelona. Universitat de Barcelona. IDIBAPS. RTA (RETICS). Barcelona, España. \\ ** Programa de abuso de sustancias, Agencia de salud pública de Cataluña, Departamento de Salud, Gobierno de Cataluña. \\ Barcelona, España.
}

\section{Resumen}

Los objetivos fueron examinar las propiedades psicométricas de la prueba de detección de consumo de alcohol, tabaco y sustancias (ASSIST) en pacientes con trastorno psiquiátrico. Un total de 202 pacientes ingresados en psiquiatría del Hospital Clínic completaron: ASSIST, Índice de gravedad de la adicción (ASI), MINIEntrevista Neuropsiquiátrica Internacional (MINI), cuestionario de identificación de los trastornos debidos al consumo de alcohol (AUDIT), Test de Fagerström (FTND), Escala de gravedad de la dependencia (SDS) y Prueba de detección de abuso de drogas (DAST). Se obtuvieron la fiabilidad y evidencia de validez de la estructura interna (análisis factorial exploratorio/confirmatorio) y de la relación con otras variables. Se encontró excelente consistencia interna en puntuaciones de riesgo total (TSI) $(\alpha=, 92$ y $\omega=, 93)$ y de cada sustancia (SSI) $(\alpha=, 88$ - ,96 y $\omega=, 89$ - ,95). La estructura interna de tabaco, alcohol y cannabis resultó en modelos unidimensionales con índices de bondad de ajuste adecuados. Las puntuaciones del ASSIST correlacionaron significativamente con: ASI $(r=, 795$ a $r=$ ,953), AUDIT $(r=, 864)$, FTND $(r=, 808)$, DAST $(r=, 831)$, SDS $(r=$ ,519) y «número de diagnósticos de abuso/dependencia» en MINIPlus (TSI: $r=, 857$ - ,862; SSI: $r=, 646$ - ,834). El análisis de curva ROC y U de Mann-Whitney mostraron evidencias de validez discriminativa. Las puntuaciones del ASSIST tienen buena fiabilidad y existen evidencias de validez para su uso en la detección del nivel de riesgo de consumo de tabaco, alcohol y sustancias en pacientes con trastorno psiquiátrico.

Palabras clave: Adicción; trastorno por consumo de alcohol; trastorno por consumo de sustancias; ASSIST; salud mental; cribado.

\begin{abstract}
The aims of this study were to examine the psychometric properties of The Alcohol, Smoking and Substance Involvement Screening Test (ASSIST) in psychiatric inpatients, due to the scarcity of screening instruments validated in this population. Patients from Hospital Clínic's psychiatric ward $(\mathrm{n}=202)$ completed: ASSIST, Addiction Severity Index (ASI), MINI-International Neuropsychiatric Interview (MINI), Alcohol Use Disorders Identification Test (AUDIT), Fagerström Test for Nicotine Dependence (FTND), Severity of Dependence Scale (SDS), and Drug Abuse Screening Test (DAST). Reliability and validity evidences based on internal structure (Exploratory and Confirmatory Factor Analyses) and on the relation to other variables were obtained. Excellent internal consistency was found for Total Substance Involvement (TSI) $(\alpha=.92$ and $\omega=.93)$ and for Specific Substance Involvement (SSI) scores $(\alpha=.88-.96$ and $\omega=.89-.95)$. Analysis of internal structure for tobacco, alcohol and cannabis subscales resulted in unidimensional models with adequate goodness-of-fit indices. ASSIST scores were significantly correlated with those of ASI $(r=.795$ to $r=.953)$, AUDIT $(r=.864)$, FTND ( $r=$ $.808)$, DAST $(r=.831)$, SDS $(r=.519)$ and with "number of diagnoses of abuse/dependence" in MINI-Plus (TSI: $r=.857$ to $r=.862$; SSI: $r=$ .646 to $r=.834$ ). Receiver operating characteristic analysis (ROC) and Mann-Whitney's U test found good discriminative validity evidences. ASSIST scores showed good reliability and there were validity evidences that support its use for identifying risk levels of tobacco, alcohol and other substance use in psychiatric patients.

Keywords: Addiction; alcohol use disorder; substance use disorder; ASSIST; mental health; screening.
\end{abstract}




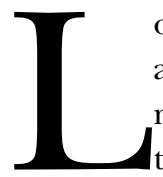

os trastornos por uso de alcohol y sustancias (TUS) afectan aproximadamente al 2,6\% de la población mundial cada año (Degenhardt et al., 2017). Los trastornos psiquiátricos están asociados con un mayor riesgo de TUS. Entre aquellos con un trastorno mental comórbido, el otro trastorno psiquiátrico a menudo precede al TUS (Degenhardt et al., 2019). Las prevalencias estimadas de comorbilidad a lo largo de la vida de los trastornos del estado de ánimo y de ansiedad con cualquier TUS son 40,9\% y 29,9\% respectivamente (Conway, Compton, Stinson y Grant, 2006) y las de trastorno bipolar y esquizofrenia con cualquier TUS superan el 40\% (Dixon, 1999; Merikangas et al., 2011).

Una baja proporción de personas con TUS recibe tratamiento por adicción (solo el $11 \%$ de aquellas con TUS el año anterior), siendo esta proporción ligeramente mayor (18\%) entre aquellas con un trastorno psiquiátrico comórbido (Harris et al., 2019). En pacientes con trastorno psiquiátrico, cuando el TUS comórbido queda sin diagnosticar y sin tratar, el curso de la enfermedad es más grave e incapacitante, y obtienen peores resultados de tratamiento que los que solo tienen una adicción (Morisano, Babor y Robaina, 2014). Para lograr un diagnóstico y tratamiento efectivos del TUS comórbido, es importante integrar el cribado en la práctica diaria en las unidades de hospitalización psiquiátrica (Crome, Bloor y Thom, 2006). Con la excepción de ASSIST, los instrumentos de cribado existentes no abarcan todas las sustancias ni son útiles en la toma de decisiones sobre el tipo de intervención más apropiada, porque la mayoría de ellos se centran en la dependencia.

La Organización Mundial de la Salud (OMS) desarrolló el ASSIST (Ali et al., 2002) para el cribado de todo tipo de sustancias. Se compone de ocho ítems (P1: uso a lo largo de la vida, P2: frecuencia de uso durante los últimos 3 meses, P3: deseo de consumir sustancias, P4: problemas de salud, sociales, económicos o legales asociados con el consumo de sustancias, P5: incumplimiento de las obligaciones propias, P6: preocupación de familiares, amigos o profesionales sobre su consumo, P7: intentos fallidos de eliminar o reducir el uso y P8: inyección de drogas a lo largo de la vida). Pueden derivarse varios dominios o puntuaciones: Puntuación de riesgo Específica para cada Sustancia (SSI) (suma de las cargas de las respuestas a los ítems 1 a 7) y la Puntuación de riesgo Total (TSI) (suma de las cargas de las respuestas a los ítems 1 a 8 de todas las sustancias), así como frecuencia, dependencia y abuso. El SSI es una puntuación para cada sustancia que determina el nivel de riesgo (bajo, moderado o alto) y la intervención más adecuada para ese nivel (ningún tratamiento, intervención breve o derivación a tratamiento de adicción, respectivamente).

La clasificación de TUS propuesta en la CIE-11 abarca diferentes niveles de uso de sustancias, desde uso nocivo único hasta conductas adictivas consolidadas, con el objetivo de facilitar el reconocimiento temprano de los problemas de salud derivados del uso de sustancias y la provisión de intervenciones de prevención y tratamiento (Bascarán, Flórez, Seijo y García, 2019). En este sentido, el ASSIST puede ser un instrumento útil para identificar diferentes niveles de riesgo en pacientes con trastorno psiquiátrico e incrementar el acceso temprano a intervenciones adecuadas.

La validación original del ASSIST (Humeniuk et al., 2008) y la validación en una muestra española (Rubio Valladolid et al., 2014) incluyeron pacientes de atención primaria y de centros de tratamiento de las adicciones. Existe un estudio de validación en población psiquiátrica que obtuvo buenos resultados pero se limitó a pacientes con un primer episodio de psicosis (Hides et al., 2009). Los objetivos del estudio fueron evaluar la fiabilidad de las puntuaciones y obtener evidencias de validez de la versión española de ASSIST para apoyar su uso en la valoración del nivel de riesgo bajo, moderado y alto del consumo de tabaco, alcohol y sustancias en pacientes con trastorno psiquiátrico hospitalizados.

Se esperó encontrar una fiabilidad similar a la reportada en estudios anteriores (Hides et al., 2009; Humeniuk et al., 2008; Rubio Valladolid et al., 2014), de entre ,89 y ,93 para la puntuación TSI y superior a ,75 para la puntuación SSI y una estructura interna unifactorial según resultados de estudios previos (Pérez-Moreno, Calzada-Álvarez, Rovira-Guardiola y Torrico Linares, 2012; Tiburcio Sainz et al., 2016). Otra hipótesis fue que las puntuaciones del ASSIST tendrían una correlación entre moderada y alta con las puntuaciones de otros instrumentos considerados de referencia en adicciones y otras variables relacionadas.

\section{Método}

\section{Participantes}

Este estudio transversal se realizó en un hospital general terciario que presta servicios especializados a una población de ingresos medios, principalmente de nacionalidad española. Los sujetos elegibles (Figura 1) incluyeron pacientes de 18 años o más hospitalizados en la unidad de hospitalización psiquiátrica de agudos del Hospital Clínic de Barcelona con sintomatología estabilizada y con fecha de alta en los siguientes cuatro días. Los criterios de exclusión fueron: 1) puntuación en el Mini Examen Cognoscitivo (Lobo, Ezquerra, Gómez, Sala y Seva, 1979) por debajo de 27; 2) diagnóstico de deterioro cognitivo significativo o retraso mental; 3) incapacidad para comunicarse debido a barrera idiomática, sordera o deficiencias visuales severas; 4) conducta agresiva; 5) confusión o déficits de memoria debido a terapia electroconvulsiva reciente; y 6) presencia de síntomas psiquiátricos agudos no estabilizados. De 224 candidatos elegibles, 13 pacientes se negaron a participar y 211 dieron su consentimiento informado. Se reclutó una muestra de conveniencia de 202 personas que completaron con éxito todas las pruebas.

La muestra $(n=202)$ consistió en 166 pacientes hospitalizados por un trastorno psiquiátrico y 36 para desintoxi- 
cación por un trastorno por uso de alcohol o sustancias. La estancia media en el hospital fue de 19,6 días $(S D=11,0)$. La edad osciló entre 19 y 84 años, con una media de 44,0 $\pm 15,5$ años. El $47 \%$ de la muestra eran hombres, el $52 \%$ soltero/a, el 32,2\% tenía empleo y el 74,3\% informó haber completado la educación secundaria o superior (véase tablas A y B en el material de apoyo para una descripción detallada de la muestra).

\section{Procedimiento}

El proceso de validación cumplió con los estándares AERA, APA y NCME (American Educational Research Association, American Psychological Association, y National Council on Measurement in Education, 2014; Muñiz y Fonseca-Pedrero, 2019) para fuentes de evidencia de validez en pruebas educativas y psicológicas. La fiabilidad de las puntuaciones se estimó mediante la consistencia interna. Se obtuvieron evidencias de validez basadas en la estructura interna y en la relación con otras variables.

Para comprobar que los ítems de la versión española del ASSIST (Rubio Valladolid et al., 2014) eran comprensibles para esta población, se evaluaron con el ASSIST a 20 pacientes hospitalizados en psiquiatría seleccionados aleatoriamente antes de iniciar el reclutamiento de la muestra. Se encontraron algunas dificultades de comprensión con la versión autoadministrada que se solucionaron cuando un psicólogo administró la prueba. Por tanto, se descartó la autoadministración. Los pacientes ingresados consecutivamente en la unidad de agudos fueron entrevistados por un psicólogo clínico con experiencia en adicciones. Se informó a los pacientes que su participación era voluntaria. El tiempo de administración de la batería de tests fue de 60 a 90 minutos y del ASSIST de 5 a 15 minutos. Los pacientes fueron asignados al Grupo de psiquiatría general (GPG) si habían sido hospitalizados debido a un trastorno psiquiátrico o al Grupo de Adicciones (GA) si habían ingresado debido a un trastorno por uso de alcohol o sustancias.

\section{Mediciones}

Este estudio utilizó el protocolo desarrollado por el grupo ASSIST de la OMS (Humeniuk et al., 2008). Se recogieron datos sociodemográficos y diagnóstico psiquiátrico de TUS realizado por un psiquiatra desconocedor de las puntuaciones ASSIST. Los participantes completaron el ASSIST V3.0 (Rubio Valladolid et al., 2014) y la siguiente batería de pruebas (Figura 1) en su versión en español:

Índice de Gravedad de la Adicción (ASI): entrevista semiestructurada para evaluar la gravedad de problemas en varias áreas (salud médica, situación laboral, aspectos legales, familiar/social, psiquiátrico, uso de alcohol y drogas) en pacientes con abuso de sustancias. El ASI-6, la versión más reciente del ASI, contiene 257 ítems. La información la proporciona el paciente mediante respuestas a preguntas cerradas y respuestas tipo Likert con un rango entre 0 y 4.
Solo se utilizó el apartado de consumo de drogas y alcohol. La consistencia interna osciló entre, 47 y ,95 y la fiabilidad test-retest osciló entre ,36 y 1 . El estudio de la estructura interna reveló un buen ajuste a una solución unidimensional para todas las escalas (Díaz-Mesa et al., 2010).

MINI-Entrevista Neuropsiquiátrica Internacional (MINI) (Ferrando et al., 1998): entrevista estructurada que evalúa los criterios diagnósticos para los trastornos psiquiátricos del DSM-IV y CIE-10. Los items tienen respuestas dicotómicas. Solo se utilizaron las secciones de consumo de drogas y alcohol. Determina la presencia o ausencia de diagnósticos de dependencia y/o abuso de alcohol y de las dos sustancias más problemáticas y si existe o no un diagnóstico actual y/o a lo largo de la vida. Los valores kappa para la fiabilidad interjueces estaban por encima de ,75 y la mayoría eran igual o superior a ,90; respecto a la fiabilidad test-retest, la mayoría de los valores estaban por encima de, 75 y solo uno por debajo de ,45 (Sheehan et al., 1998).

Escala de gravedad de la dependencia (SDS) (González-Sáiz y Carulla-Salvador, 1998): escala de cinco ítems centrados en los aspectos psicológicos de la dependencia de sustancias que mide la gravedad del uso de sustancias. Cada ítem se puntúa en una escala de 4 puntos (0 a 3). Se encontraron coeficientes de fiabilidad adecuados para todas las escalas de dependencia de sustancias $(\alpha=, 737-, 877$; test-retest $r=, 796-, 952)$. Se halló una baja consistencia interna para las escalas de abuso $(\alpha=, 329-, 694)$, y coeficientes test-retest adecuados en alcohol, cocaína y heroína (test-retest $r=, 708$ - ,902) (Vélez-Moreno et al., 2015).

Cuestionario de Identificación de los Trastornos debidos al Consumo de Alcohol (AUDIT): prueba de cribado para identificar el consumo de riesgo y perjudicial y la dependencia del alcohol. Tiene diez ítems con tres o cuatro opciones de respuesta. La fiabilidad interna medida por el coeficiente alfa de Cronbach fue de ,86 y el coeficiente de correlación test-retest fue de ,90 (Rubio Valladolid, Bermejo Vicedo, Caballero Sánchez-Serrano y Santo-Domingo Carrasco, 1998).

Prueba de detección de abuso de drogas (DAST-10): prueba autoadministrada que tiene 10 ítems con respuesta dicotómica, y proporciona un índice cuantitativo de la medida en que se sufren problemas de abuso de drogas midiendo una dimensión subyacente de dependencia. Tiene una consistencia interna alta $(\alpha=, 89)$. El análisis factorial exploratorio en su versión española extrajo dos componentes que explicaron el $62,18 \%$ de la varianza. Los puntos de corte $(\geq 3)$ mostraron un alto grado de concordancia con los criterios diagnósticos de DSM-IV TR ( clasificando correctamente a más del $90 \%$ de los sujetos evaluados (Pérez-Gálvez, García-Fernández, de Vicente-Manzanaro, Oliveras-Valenzuela y Lahoz-Lafuente, 2010).

Test de Fagerström de dependencia de la nicotina (FTNDT): cuestionario de autoinforme con seis ítems, diseñado para medir la gravedad de la dependencia de nicotina. Los ítems tienen entre dos y cuatro opciones de respuesta y pro- 


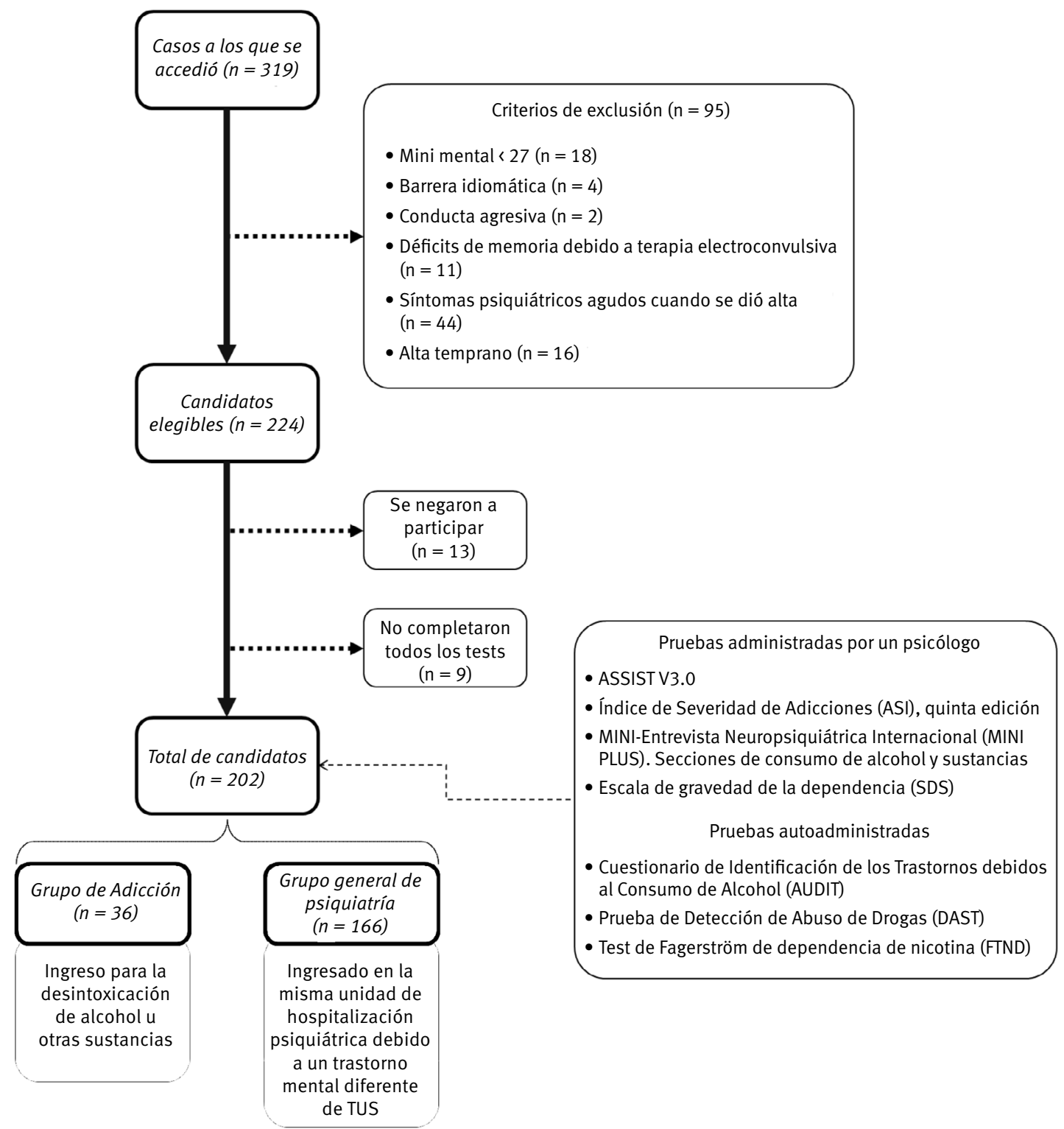

Figura 1. Diagrama de flujo del reclutamiento de pacientes y la batería de pruebas aplicada a la muestra de candidatos $(n=202)$.

porcionan una puntuación total que oscila entre 0 y 10 . El coeficiente alfa de Cronbach para el Test de Fagerstrom fue de ,66 (Becoña y Vázquez, 1998).

\section{Análisis de datos}

Se utilizó el paquete estadístico SPSS (IBM Corp. Released, 2019) y el software estadístico R (R Core Team, 2020).

Solo se incluyeron los participantes que respondieron al ASSIST y a la batería completa de pruebas, por lo que no se requirió ningún procedimiento de imputación de valores perdidos. La prueba de Kolmogorov-Smirnov fue utilizada para comprobar la distribución normal de los datos. Dado que los ítems del ASSIST no siguieron una distribución normal, se utilizaron pruebas no paramétricas. Se utilizó la prueba U de Mann-Whitney para comparar medianas y la prueba de Spearman para los análisis de correlación. Un valor de $\mathrm{p}$ $<, 001$ se consideró estadísticamente significativo. Los ítems del ASSIST se describieron utilizando media, desviación estándar, mediana, rango intercuartílico, asimetría y curtosis. Los índices de discriminación de los ítems se calcularon mediante la correlación ítem-total corregida considerada adecuada por encima de ,30 (Nunnally y Bernstein, 1994).

La evidencia de validez basada en la estructura interna se evaluó mediante análisis factorial. La estructura dimensional del ASSIST se analizó dividiendo aleatoriamente la muestra en dos mitades. La primera de las dos mitades se utilizó para realizar un análisis factorial exploratorio (AFE) para detectar la estructura latente de la subescala de cada sustancia mediante el método de máxima verosimilitud con rotación Varimax.

Con la segunda mitad de la muestra, se exploró la estructura resultante del análisis anterior mediante un análisis factorial confirmatorio (AFC) con el procedimiento de máxima verosimilitud. Para comprobar el ajuste global del 
modelo se analizaron la prueba de bondad de ajuste de $\chi^{2}$, el Indice comparativo de ajuste (CFI) y el valor Raiz media cuadrática del Error de Aproximación (RMSEA). Los valores RMSEA < ,06 y CFI > ,95 indican un buen ajuste (Hu y Bentler, 1999).

Para evaluar la fiabilidad de las puntuaciones, se midió la consistencia interna de las puntuaciones TSI y SSI según el alfa de Cronbach (Cronbach, 1951). Además, de- bido a las limitaciones del coeficiente alfa de Cronbach, también se calculó el omega de McDonald (Dunn, Baguley y Brunsden, 2014).

Se examinó la evidencia de validez en función de las relaciones con otras variables. La correlación de Spearman se utilizó para comparar las puntuaciones de los dominios ASSIST con las puntuaciones de otros instrumentos administrados simultáneamente y considerados de referencia en adicciones (Figura 1). El GA se dividió en dos grupos según la presencia o ausencia de un diagnóstico de dependencia realizado por un psiquiatra desconocedor de las puntuaciones ASSIST. Las puntuaciones SSI del ASSIST en ambos grupos se compararon mediante la prueba U de Mann-Whitney.

Además, los dominios ASSIST que miden el abuso y la dependencia se compararon con las puntuaciones derivadas «número total de diagnósticos de abuso»y «número total de diagnósticos de dependencia» en el MINI utilizando la correlación de Spearman. Los dominios ASSIST «Consumo de sustancias a lo largo de la vida» y TSI se compararon con los ítems del ASI: «número de tratamientos previos por abuso de alcohol o sustancias» $\mathrm{y}$ «gasto económico en alcohol o drogas en los últimos tres meses», considerados como medidas indirectas de abuso o dependencia.

Las evidencias de validez discriminante se probaron comparando las puntuaciones del ASSIST de los siguientes grupos: bajo riesgo (pacientes de GPG sin un diagnóstico de abuso o dependencia), riesgo moderado (pacientes del GPG con diagnóstico de abuso o dependencia según el MINI) y alto riesgo (pacientes del GA ingresados por un TUS actual) mediante la prueba U de Mann-Whitney. El análisis ROC (Característica Operativa del Receptor) y la sensibilidad y especificidad de las puntuaciones de corte se calcularon cuando fue posible y se compararon con la sensibilidad y especificidad obtenidas utilizando las puntuaciones de corte sugeridas por la OMS.

Las estimaciones del tamaño del efecto se calcularon mediante la d de Cohen (Cohen, 1988).

\section{Cuestiones éticas}

El protocolo fue aprobado por el Comité de Ética del Hospital Clínic (CEIm, número 2011/6516), de acuerdo con la Declaración de Helsinki (World Medical Association Declaration of Helsinki, 2013) y la Ley 14/2007, de 3 de julio, de Investigación biomédica. Se garantizó el anonimato de los participantes y la confidencialidad de los datos.

\section{Resultados}

La Tabla 1 muestra la descripción de los primeros siete ítems de ASSIST. Los índices de correlación ítem-total corregidos superaron ,40, excepto $\mathrm{P} 1$ y $\mathrm{P} 2$ para alcohol, lo que significa que los ítems muestran una buena discriminación.

\section{Evidencias de estructura interna de ASSIST}

El AFE para las subescalas de tabaco, alcohol, cannabis, cocaína, anfetaminas y sedantes resultó en una estructura unifactorial para cada una (con autovalores que oscilaron entre 2,9 y 4,8) que explicaron entre $57,3 \%$ y $80,3 \%$ de la varianza. Todas las cargas factoriales fueron mayores de ,5 en todas las preguntas. Las otras subescalas de sustancias no tenían datos suficientes para realizar un AFE. La Tabla 2 detalla los autovalores, los porcentajes de varianza explicada, las cargas factoriales y el índice de Kaiser-Meyer-Olkin (KMO).

La Figura 2 muestra los resultados del AFC. Los análisis de las subescalas de sustancias se basaron en los resultados del AFE, sugiriendo escalas unidimensionales. Para tabaco, utilizando el método de los multiplicadores de Lagrange, el mejor ajuste del modelo se obtuvo al correlacionar las preguntas 6 y $7(r=, 246)\left(\chi^{2}(3)=5,298, p=, 258\right)(\mathrm{CFI}=, 995$, RMSEA $=, 059$, RMSEA 90\% IC $=, 000-, 177)$.

Se aplicó el mismo procedimiento a la subescala alcohol. La pregunta 2 estaba relacionada con la pregunta 6 ( $\mathrm{r}=$ ,284) y la pregunta $7(r=, 241)\left(\chi^{2}(7)=9,133, p=, 243\right)(\mathrm{CFI}$ $=, 996$, RMSEA $=, 058$, RMSEA 90\% IC $=, 000-, 148)$.

Para la subescala cannabis, la pregunta 2 estaba relacionada con las preguntas $3(r=, 541)$ y $6(r=, 372)$ y la pregunta 7 estaba relacionada con las preguntas $6(r=, 250)$ y $5(r$ $=, 77)\left(\chi^{2}(5)=5,728, p=, 334\right)(\mathrm{CFI}=, 999, \mathrm{RMSEA}=, 040$, RMSEA $90 \%$ IC $=, 000-, 155)$. Las otras subescalas de sustancias no obtuvieron un ajuste adecuado en el análisis.

\section{Fiabilidad de las puntuaciones ASSIST}

La consistencia interna, estimada mediante el coeficiente $\alpha$ de Cronbach, era de ,92 para la puntuación TSI con un rango entre ,88 y ,96 para las puntuaciones SSI. El Omega de McDonald era de ,93 para la puntuación TSI y entre ,89 y ,96 para las puntuaciones SSI (la Tabla C en el material de apoyo muestra resultados detallados).

\section{Evidencias de validez basadas en la relación con otras variables}

Se encontraron correlaciones positivas significativas entre el ASSIST y los instrumentos de referencia en adicciones, tal como se resume en la Tabla 3 .

Las puntuaciones SSI de los sujetos con un diagnóstico MINI de «abuso o dependencia» fueron significativamente más altas que las puntuaciones de aquellos sujetos sin diagnóstico. Las puntuaciones SSI para aquellos participantes con un diagnóstico de dependencia actual realizado por un psiquiatra, fueron significativamente más altas que las de los 
Tabla 1. Descripción de los ítems de ASSIST.

\begin{tabular}{|c|c|c|c|c|c|c|c|}
\hline & $\mathbf{P}_{1}$ & $\mathbf{P}_{2}$ & $\mathrm{P}_{3}$ & $\mathbf{P 4}$ & $\mathrm{P}_{5}$ & P6 & $\mathrm{P}_{7}$ \\
\hline \multicolumn{8}{|l|}{ Tabaco } \\
\hline Media (SD) & $2,58(1,04)$ & $3,39(2,93)$ & $3,3(2,95)$ & $1,57(2,71)$ & & $2,79(2,7)$ & $1,56(2,12)$ \\
\hline Mediana $(R / Q)$ & $3(3-3)$ & $6(0-6)$ & $6(0-6)$ & $0(0-4)$ & & $3(0-6)$ & $0(0-3)$ \\
\hline Asimetría & $>2,47$ & $-1,91$ & $-1,95$ &,- 37 & & $-1,76$ &,- 34 \\
\hline Curtosis & $-2,11$ &,- 27 &,- 22 & 1,22 & & ,14 & 1,00 \\
\hline Îndice de discriminación & ,456 & ,868 & ,873 &, 537 & & ,753 &, 597 \\
\hline \multicolumn{8}{|l|}{ Alcohol } \\
\hline Media (SD) & $2,97(, 3)$ & $2,65(1,94)$ & $1,1(2,16)$ & $1,4(2,58)$ &, $97(2,43)$ & $1,57(2,42)$ & ,91 (1,97) \\
\hline Mediana (RIQ) & $3(3-3)$ & $3(0-4)$ & $0(0-0)$ & $0(0-0)$ & $0(0-0)$ & $0(0-3)$ & $0(0-0)$ \\
\hline Asimetría & 98,46 &,- 97 &, 57 & ,03 & 2,81 &,- 60 & 2,16 \\
\hline Curtosis & $-9,97$ & 00 & 1,54 & 1,38 & 2,16 & 1,07 & 1,94 \\
\hline Îndice de discriminación & ,107 & ,615 & ,853 & ,898 &, 761 & ,778 & ,806 \\
\hline \multicolumn{8}{|l|}{ Cánnabis } \\
\hline Media (SD) & $1,78(1,48)$ & $1,05(2,07)$ & ,95 (2,09) & $1,06(2,34)$ & , $9(2,37)$ & $1,25(2,19)$ & ,65 (1,67) \\
\hline Mediana $(R / Q)$ & $3(0-3)$ & $0(0-0)$ & $0(0-0)$ & $0(0-0)$ & $0(0-0)$ & $0(0-3)$ & $0(0-0)$ \\
\hline Asimetría & $-1,87$ & 1,14 & 1,45 & 1,40 & 3,51 & ,39 & 4,8 \\
\hline Curtosis &,- 39 & 1,67 & 1,82 & 1,81 & 2,31 & 1,42 & 2,47 \\
\hline Îndice de discriminación &, 527 & 912 & ,891 & ,893 &, 802 & ,826 & ,708 \\
\hline \multicolumn{8}{|l|}{ Cocaína } \\
\hline Media (SD) & $1,01(1,42)$ & ,3 $(1,04)$ &, $35(1,27)$ &, $34(1,33)$ &, $37(1,55)$ & ,49 $(1,46)$ & ,31 (1,17) \\
\hline Mediana $(R / Q)$ & $0(0-3)$ & $0(0-0)$ & $0(0-0)$ & $0(0-0)$ & $0(0-0)$ & $0(0-0)$ & $0(0-0)$ \\
\hline Asimetría & $-1,53$ & 12,97 & 10,32 & 12,98 & 15,49 & 7,94 & 15,13 \\
\hline Curtosis &, 70 & 3,63 & 3,45 & 3,79 & 4,11 & 3,00 & 3,95 \\
\hline Índice de discriminación &, 583 & ,857 &, 85 & ,86 & ,795 & ,792 & ,808 \\
\hline \multicolumn{8}{|l|}{ Anfetaminas } \\
\hline Media (SD) &, $65(1,24)$ & ,14 (,78) & , 1(,74) & ,14 (,9) & ,14 (,98) &, $16(, 86)$ &, $06(, 51)$ \\
\hline Mediana $(R / Q)$ & $0(0-0)$ & $0(0-0)$ & $0(0-0)$ & $0(0-0)$ & $0(0-0)$ & $0(0-0)$ & $0(0-0)$ \\
\hline Asimetría &,- 10 & 37,94 & 51,29 & 44,69 & 48,79 & 33,12 & 98,69 \\
\hline Curtosis & 1,38 & 6,06 & 7,20 & 6,67 & 7,06 & 5,67 & 9,59 \\
\hline Índice de discriminación &, 567 &, 814 & ,734 &, 767 &, 760 &, 714 &, 509 \\
\hline \multicolumn{8}{|l|}{ Sedantes } \\
\hline Media (SD) & ,67 (1,25) &, $75(1,86)$ & ,7 (1,89) &, $78(2,11)$ &, $63(2,06)$ & ,64 (1,77) &, $43(1,45)$ \\
\hline Mediana $(R / Q)$ & $0(0-0)$ & $0(0-0)$ & $0(0-0)$ & $0(0-0)$ & $0(0-0)$ & $0(0-0)$ & $0(0-0)$ \\
\hline Asimetría &,- 20 & 3,39 & 3,66 & 4,08 & 7,44 & 4,88 & 9,65 \\
\hline Curtosis & 1,34 & 2,25 & 2,35 & 2,43 & 3,03 & 2,56 & 3,32 \\
\hline Índice de discriminación & ,789 & ,932 & 0,93 & ,901 & ,794 &, 815 & ,774 \\
\hline \multicolumn{8}{|l|}{ Opioides } \\
\hline Media (SD) & ,28 (,88) & ,12(,76) & ,13 (,82) & ,13 (,92) & ,11(,9) & ,18 (,93) & , 19 (0,99) \\
\hline Mediana $(R / Q)$ & $0(0-0)$ & $0(0-0)$ & $0(0-0)$ & $0(0-0)$ & $0(0-0)$ & $0(0-0)$ & $0(0-0)$ \\
\hline Asimetría & 5,91 & 43,33 & 39,53 & 49,75 & 68,00 & 30,28 & 27,52 \\
\hline Curtosis & 2,80 & 6,57 & 6,36 & 7,12 & 8,27 & 5,50 & 5,31 \\
\hline Índice de discriminación &, 650 & ,907 & ,885 & ,814 & ,782 &, 826 & ,879 \\
\hline
\end{tabular}

Nota. No hay datos suficientes para inhalantes y alucinógenos.

Tabla 2. Análisis factorial exploratorio.

\begin{tabular}{|c|c|c|c|c|c|c|c|c|}
\hline \multirow{2}{*}{ Subescala } & \multirow{2}{*}{ Número de factores } & \multirow{2}{*}{ Eigenvalor } & \multirow{2}{*}{ Varianza explicada } & \multirow{2}{*}{ Cargas factoriales } & \multirow{2}{*}{ KMO } & \multicolumn{3}{|c|}{$\chi^{2}$ de Barret } \\
\hline & & & & & & $\chi^{2}$ & $d f$ & $p$ \\
\hline Tabaco & 1 & 2,9 & $57,3 \%$ & $>, 50$ & ,78 & 314,88 & 10 & $<, 001$ \\
\hline Alcohol & 1 & 4,1 & $68,0 \%$ & $>, 70$ & ,88 & 506,99 & 15 & $<, 001$ \\
\hline Cánnabis & 1 & 4,8 & $80,3 \%$ & $>, 80$ & ,85 & 515,14 & 15 & $<, 001$ \\
\hline Cocaína & 1 & 4,6 & $76,6 \%$ & $>, 70$ & ,86 & 317,05 & 15 & $<, 001$ \\
\hline Anfetaminas & 1 & 4,2 & $70,3 \%$ & $>, 50$ & ,84 & 171,68 & 15 & $<, 001$ \\
\hline Sedantes & 1 & 3,6 & $59,7 \%$ & $>, 60$ & , 80 & 113,89 & 15 & $<, 001$ \\
\hline
\end{tabular}

Nota. $\mathrm{KMO}=$ Kaiser-Meyer-Olkin. 

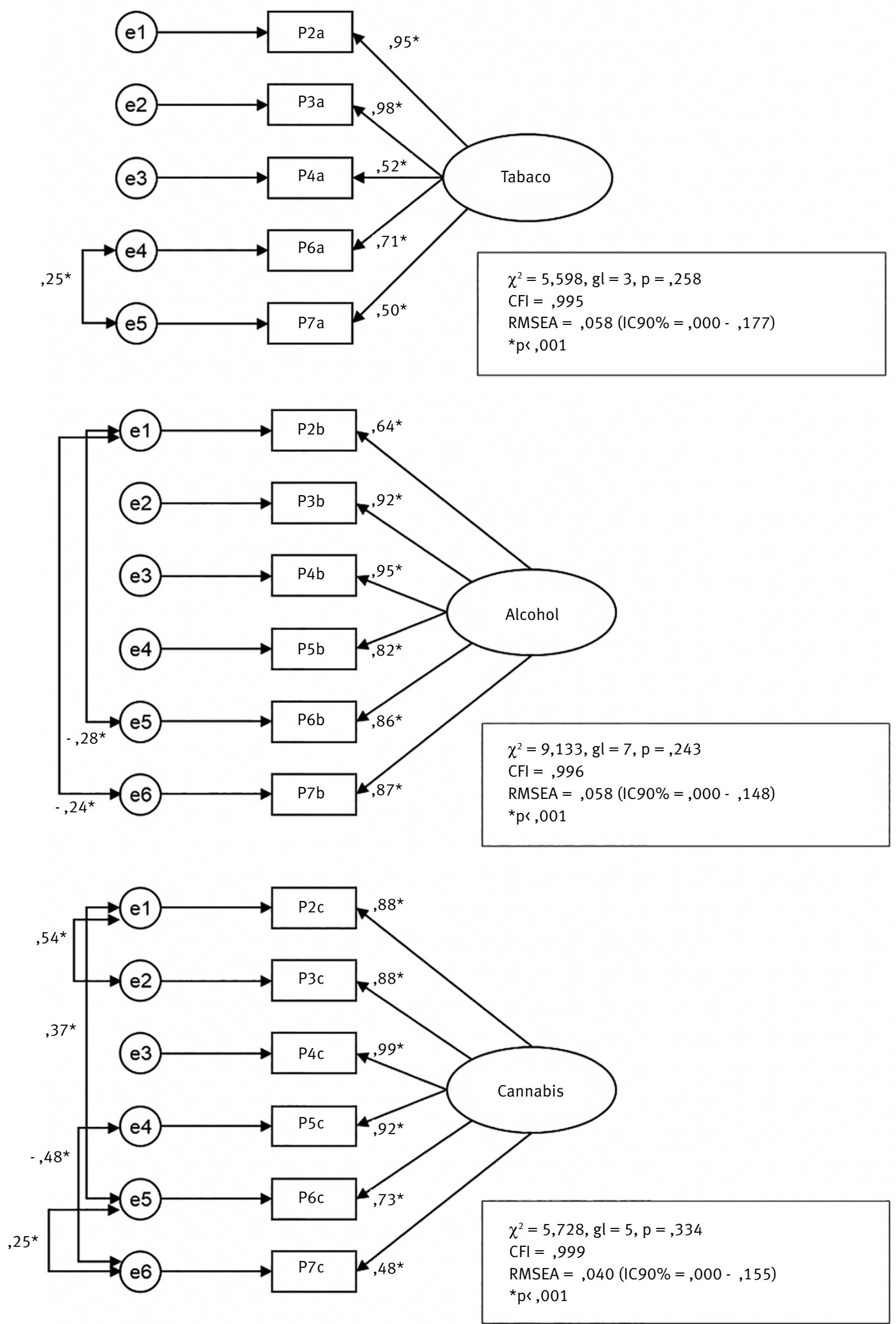

Figura 2. Análisis factorial confirmatorio para las subescalas de tabaco, alcohol y cannabis de ASSIST.

sujetos sin diagnóstico para tabaco, alcohol, cocaína, sedantes y opioides en el GA (ver Tabla 4).

Respecto de las evidencias de validez discriminativa (ver Tabla 5), hubo diferencias significativas en las puntuaciones SSI entre los grupos de riesgo bajo y moderado para al- cohol, cannabis, cocaína, anfetaminas y sedantes $(\mathrm{p}<, 001)$ y entre los grupos de riesgo moderado y alto para alcohol. No hubo diferencias significativas entre los grupos de riesgo moderado y alto (dependencia) en SSI para cannabis, cocaína y sedantes. 
No se pudo investigar la discriminación entre riesgo moderado y alto para anfetaminas debido a la ausencia de sujetos con diagnóstico de dependencia (riesgo alto) en el GA. Para inhalantes, opioides y alucinógenos, no pudieron calcularse la curva ROC y la U de Mann-Whitney debido a la insuficiencia de datos.
Según el análisis ROC, el ASSIST puede discriminar mejor entre riesgo bajo y moderado que entre riesgo moderado y alto. El área bajo la curva es más alta para las comparaciones entre riesgo bajo y moderado en todas las sustancias (puntuaciones de corte entre 1,50 y 33,5, área bajo la curva (AUC) entre ,386 y ,991).

Tabla 3. Evidencias de validez en base a la relación con otras variables.

Correlación entre los dominios ASSIST y los instrumentos de referencia

r de Spearman

,709

,519

,831

,808

,864

,862

TSI y MINI más «número de diagnósticos» de abuso o dependencia actual o a lo largo de la vida al alcohol y un máximo de dos sustancias

TSI Ilícito y MINI más «número de diagnósticos» de abuso o dependencia actual o a lo largo de la vida al alcohol y un máximo de dos sustancias

SSI para todas las sustancias y MINI más «número de diagnósticos»

, $646-, 834>, 001$

ASSIST «Dependencia» para sustancias ilícitas y DAST

ASSIST «Abuso» para sustancias ilícitas y DAST

ASSIST «Frecuencia total y actual» para todas las sustancias y ASI «Frecuencia de uso de cada sustancia» ASSIST «Dependencia» para todas las sustancias y MINI más «número total de diagnósticos de dependencia» ASSIST «Dependencia» para todas las sustancias y MINI más «número total de diagnósticos de dependencia» ASSIST «Abuso» para todas las sustancias y MINI más «número total de diagnósticos de abuso» ASSIST «Abuso» para todas las sustancias ilícitas y MINI más «número total de diagnósticos de abuso» ASSIST «Uso de sustancias a lo largo de la vida» y ASI «Uso de sustancias a lo largo de la vida» ASSIST «Uso de sustancias a lo largo de la vida» y ASI «Número de tratamientos anteriores» TSI y ASI «Gasto en alcohol o drogas en los últimos tres meses»

$\begin{array}{cc}, 821 & >, 001 \\ , 826 & >, 001 \\ , 795-, 953 & >, 001 \\ , 795 & >, 001 \\ , 825 & >, 001 \\ , 842 & >, 001 \\ , 837 & >, 001 \\ , 430 & >, 001 \\ , 460 & >, 001 \\ , 722 & >, 001\end{array}$

Tabla 4. Puntuaciones de riesgo Específicas para cada sustancia (SSI) según la presencia o no de criterios para un diagnóstico de abuso o dependencia actual o a lo largo de la vida según MINI, y según diagnóstico psiquiátrico independiente (IPD) de dependencia en GA.

\begin{tabular}{|c|c|c|c|c|c|c|}
\hline \multirow{2}{*}{ Puntuación en SSI } & \multirow{2}{*}{$\frac{\text { Abuso o dependencia actual }}{\text { Puntuación media }}$} & \multirow{2}{*}{$\frac{\text { Ausencia de abuso o dependencia }}{\text { Puntuación media }}$} & \multicolumn{3}{|c|}{ Prueba U de Mann-Whitney } & \multirow{2}{*}{$d$ de Cohen } \\
\hline & & & $\boldsymbol{U}$ & $\boldsymbol{z}$ & $p$ & \\
\hline & \multicolumn{6}{|c|}{ Diagnóstico de abuso o dependencia actual o a lo largo de la vida según MINI (n=202) } \\
\hline Alcohol & 149,49 & 68,70 & 984,5 & $-9,74$ & $<, 001$ & 1,00 \\
\hline Cánnabis & 166,60 & 78,93 & 515,0 & $-11,41$ & $<, 001$ & 69 \\
\hline Cocaína & 161,54 & 93,02 & 711,5 & $-9,00$ & $<, 001$ & ,40 \\
\hline Anfetaminas & 154,64 & 99,59 & 310,5 & $-5,98$ & $<, 001$ &, 24 \\
\hline Sedantes & 182,28 & 90,09 & 193,0 & $-11,33$ & $<, 001$ &, 53 \\
\hline \multirow[t]{2}{*}{ Opioides } & 182,00 & 99,04 & 105,0 & $-9,11$ & $<, 001$ &, 25 \\
\hline & \multicolumn{6}{|c|}{$\operatorname{IPD}(n=36)$} \\
\hline Tabaco & 21,29 & 12,92 & 77,0 & $-2,26$ &, 024 & 2,68 \\
\hline Alcohol & 23,46 & 8,58 & 25,0 & $-4,00$ & $<, 001$ & 2,51 \\
\hline Cánnabis & 33,00 & 17,65 & 5,0 & $-2,54$ &, 038 &, 60 \\
\hline Cocaína & 32,90 & 16,18 & 5,5 & $-4,17$ & $<, 001$ & ,72 \\
\hline Sedantes & 29,94 & 15,23 & 20,5 & $-3,96$ & $<, 001$ & ,97 \\
\hline Opioides & 34,00 & 16,00 & 0 & $-5,90$ & $<, 001$ &, 50 \\
\hline
\end{tabular}


Tabla 5. Discriminación entre riesgo bajo y moderado y riesgo moderado y alto con la prueba U de Mann-Whitney y el Análisis ROC (Característica Operativa del Receptor).

\begin{tabular}{|c|c|c|c|c|c|c|c|c|c|c|c|}
\hline \multirow{2}{*}{$\begin{array}{l}\text { Nivel de riesgo } \\
\text { (n) }\end{array}$} & \multirow{2}{*}{$\begin{array}{l}\text { ROC } \\
\text { (AUC) }\end{array}$} & \multicolumn{2}{|c|}{ ROC } & \multirow{2}{*}{$\begin{array}{l}\text { ASSIST punto } \\
\text { de corte }\end{array}$} & \multirow{2}{*}{$\begin{array}{c}\text { OMS punto } \\
\text { de corte }\end{array}$} & \multicolumn{2}{|c|}{ ROC OMS } & \multicolumn{4}{|c|}{ Prueba U de Mann-Whitney } \\
\hline & & Sensibilidad & Especificidad & & & Sensibilidad & Especificidad & $\boldsymbol{U}$ & $z$ & $p$ & $d$ \\
\hline \multicolumn{12}{|c|}{ SSI Alcohol } \\
\hline $\begin{array}{l}\text { Bajo (141) / } \\
\text { Moderado (24) }\end{array}$ & ,946 & $83,3 \%$ & $86,5 \%$ & 5,50 & 11 & $66,7 \%$ & $99,3 \%$ & 183,0 & $-7,08$ & $<, 001$ & 1,95 \\
\hline $\begin{array}{l}\text { Moderado (24) / } \\
\text { Alto (24) }\end{array}$ &, 895 & $79,2 \%$ & $75,0 \%$ & 27,50 & 27 & $87,5 \%$ & $70,8 \%$ & 60,5 & $-4,70$ & $<, 001$ & 1,66 \\
\hline \multicolumn{12}{|c|}{ SSI cannabis } \\
\hline $\begin{array}{l}\text { Bajo (132) / } \\
\text { Moderado (33) }\end{array}$ & 991 & $97,0 \%$ & $97,0 \%$ & 7,50 & 4 & $100 \%$ & $94,7 \%$ & 40,5 & $-10,57$ & $<, 001$ & 3,94 \\
\hline $\begin{array}{l}\text { Moderado (33) / } \\
\text { Alto (2) }\end{array}$ &, 386 & $50,0 \%$ & $63,6 \%$ & 32,50 & 27 & $50 \%$ & $36,4 \%$ & 25,5 &,- 54 & $=, 61$ & ,49 \\
\hline \multicolumn{12}{|c|}{ SSI Cocaína } \\
\hline $\begin{array}{l}\text { Bajo (159) / } \\
\text { Moderado (9) }\end{array}$ & ,932 & $88,9 \%$ & $92,9 \%$ & 1,50 & 4 & $77,8 \%$ & $97,4 \%$ & 95,0 & $-7,86$ & $<, 001$ & 1,87 \\
\hline $\begin{array}{l}\text { Moderado (9) / } \\
\text { Alto (4) }\end{array}$ &, 833 & $75,0 \%$ & $77,8 \%$ & 31,50 & 27 & $100 \%$ & $66,7 \%$ & 6,0 & $-1,86$ & $=, 06$ & 1,38 \\
\hline \multicolumn{12}{|c|}{ SSI Anfetaminas } \\
\hline $\begin{array}{l}\text { Bajo (162) / } \\
\text { Moderado (3) }\end{array}$ & ,988 & $100 \%$ & $96,9 \%$ & 1,50 & 4 & $66,7 \%$ & $98,1 \%$ & 6,0 & $-7,77$ & $<, 001$ & 1,80 \\
\hline $\begin{array}{l}\text { Moderado (3) / } \\
\text { Alto (0) }\end{array}$ & $\mathrm{n} / \mathrm{a}$ & $\mathrm{n} / \mathrm{a}$ & $\mathrm{n} / \mathrm{a}$ & $\mathrm{n} / \mathrm{a}$ & 27 & $\mathrm{n} / \mathrm{a}$ & $\mathrm{n} / \mathrm{a}$ & $\mathrm{n} / \mathrm{a}$ & $\mathrm{n} / \mathrm{a}$ & $\mathrm{n} / \mathrm{a}$ & $\mathrm{n} / \mathrm{a}$ \\
\hline \multicolumn{12}{|c|}{ SSI Sedantes } \\
\hline $\begin{array}{l}\text { Bajo (154) / } \\
\text { Moderado (11) }\end{array}$ & 1 & $100 \%$ & $94,2 \%$ & 1,50 & 4 & $100 \%$ & $96,1 \%$ & , 0 & $-9,76$ & $<, 001$ & 7,32 \\
\hline $\begin{array}{l}\text { Moderado (11) / } \\
\text { Alto (9) }\end{array}$ &, 606 & $66,7 \%$ & $81,8 \%$ & 33,5 & 27 & $66,7 \%$ & $27,3 \%$ & 39,0 &,- 80 & $=, 42$ & ,02 \\
\hline
\end{tabular}

Nota. ROC OMS: Valores de sensibilidad y especificidad al usar los puntos de corte de ASSIST propuestos por la OMS.

\section{Discusión}

Este estudio tuvo como objetivo examinar la fiabilidad y obtener evidencias de validez del ASSIST en pacientes con trastorno psiquiátrico debido a la escasez de datos sobre su uso en esta población. Esta investigación encontró que el ASSIST tiene buenas propiedades psicométricas para medir diferentes niveles de riesgo de uso de sustancias en esta población. Se hallaron ítems con discriminación adecuada, evidencias de estructura interna unidimensional para tabaco, alcohol y cannabis, buena consistencia interna y evidencias de validez basadas en las relaciones con otros instrumentos (SDS, DAST, FTND, AUDIT, MINI, ASI). El AFE y AFC mostraron un modelo unidimensional para tabaco, alcohol, y cannabis que sugiere que la puntuación SSI obtenida para estas sustancias tiene respaldo empírico en esta muestra. Otros estudios informaron del mismo resultado para tabaco y alcohol en estudiantes universitarios (Tiburcio Sainz et al., 2016) y para cocaína en una muestra de consumidores de cocaína (Pérez-Moreno et al., 2012). Los valores de consistencia interna, que fueron entre, 88 y, 96 , fueron similares a los reportados por estudios previos (Hides et al., 2009; Humeniuk et al., 2008; Rubio Valladolid et al., 2014).
Las puntuaciones SSI fueron significativamente más altas para aquellos pacientes con un diagnóstico en el MINI de abuso o dependencia, lo que muestra que las puntuaciones SSI reflejan el uso problemático de sustancias.

El análisis ROC y la prueba U de Mann-Whitney mostraron evidencias de buena validez discriminativa, encontrando diferencias significativas especialmente entre los grupos de riesgo bajo y moderado para alcohol, cannabis, cocaína, anfetaminas y sedantes. El AUC mostró excelentes resultados (AUC > ,90). También se encontró una buena validez discriminativa (AUC > ,80) entre riesgo moderado (uso problemático) y alto riesgo (dependencia) para alcohol. De manera similar a los estudios previos (Humeniuk et al., 2008), el ASSIST discrimina con mayor eficacia entre riesgo bajo y moderado que entre riesgo moderado y alto, sin diferencias para sedantes entre los grupos de riesgo moderado y alto.

Los puntos de corte óptimos para riesgo moderado obtenidos en este estudio para alcohol, cocaína, anfetaminas y sedantes son comparables a los establecidos en el estudio de validación multicentrico (Humeniuk et al., 2008), mientras que en el estudio de validación de la versión española ( $\mathrm{Ru}-$ bio Valladolid et al., 2014) los puntos de corte para estas sustancias fueron un poco más altos. Los puntos de corte para 
Materiales de apoyo

Tabla A. Características sociodemográficas de la muestra y diferencias entre grupos con la te Student para viables continuos y chicuadrado para variables categóricas.

\begin{tabular}{|c|c|c|c|c|}
\hline & Total $\mathbf{n}=\mathbf{2 0 2}$ & $\begin{array}{c}\text { Grupo de adicción } \\
n=36\end{array}$ & $\begin{array}{l}\text { Grupo de psiquiatría } \\
\text { general } n=166\end{array}$ & Diferencias medias \\
\hline Edad media en años (SD) & $44,0(15,5)$ & $48,2(12,4)$ & $43,1(16,0)$ & $\begin{array}{c}t=1,818 \\
g l=200 \\
p<, 071 \\
d \text { de Cohen }=, 34\end{array}$ \\
\hline Mujer & 53,0 & 63,9 & 50,6 & $\begin{array}{c}\chi^{2}=2,09 \\
g l=1 \\
p<, 148\end{array}$ \\
\hline \multicolumn{5}{|l|}{ Estado civil (\%) } \\
\hline $\begin{array}{l}\text { Casado/a-Cohabitando } \\
\text { Separado/a-Divorciado/a } \\
\text { Viudo/a } \\
\text { Nunca casado/o }\end{array}$ & $\begin{array}{c}32,7 \\
11,9 \\
3,5 \\
52,0\end{array}$ & $\begin{array}{c}44,4 \\
11,1 \\
2,8 \\
41,7\end{array}$ & $\begin{array}{c}30,1 \\
12,1 \\
3,6 \\
54,2\end{array}$ & $\begin{array}{c}\chi^{2}=5,332 \\
g l=5 \\
p<, 377\end{array}$ \\
\hline $\begin{array}{l}\text { Tipo de vivienda (\%) } \\
\text { Vivienda propia o familiar } \\
\text { Alquiler de vivienda o habitación }\end{array}$ & $\begin{array}{l}63,4 \\
36,6\end{array}$ & $\begin{array}{l}63,9 \\
36,1\end{array}$ & $\begin{array}{l}63,3 \\
36,8\end{array}$ & $\begin{array}{l}\chi^{2}=, 290 \\
g l=3 \\
p<, 962\end{array}$ \\
\hline $\begin{array}{l}\text { Grupo étnico (\%) } \\
\text { Caucásica/blanca } \\
\text { Afromericano/asiático/hispano }\end{array}$ & $\begin{array}{c}94,6 \\
5,5\end{array}$ & $\begin{array}{c}94,4 \\
5,6\end{array}$ & $\begin{array}{c}94,6 \\
5,4\end{array}$ & $\begin{array}{c}\chi^{2}=, 932 \\
g l=3 \\
p<, 818\end{array}$ \\
\hline \multicolumn{5}{|l|}{ Situación laboral } \\
\hline $\begin{array}{l}\text { Empleado/a } \\
\text { Sin trabajar debido a baja médica } \\
\text { Desempleado/a } \\
\text { Discapacidad } \\
\text { Otro (Retirado/a, Estudiante o en casa) }\end{array}$ & $\begin{array}{c}32,2 \\
4,5 \\
24,8 \\
23,3 \\
15,4\end{array}$ & $\begin{array}{c}33,3 \\
5,6 \\
19,4 \\
30,6 \\
11,1\end{array}$ & $\begin{array}{c}31,9 \\
4,2 \\
25,9 \\
21,7 \\
16,3\end{array}$ & $\begin{array}{c}\chi^{2}=7,360 \\
g l=8 \\
p<, 498\end{array}$ \\
\hline $\begin{array}{l}\text { Nivel de estudios (\%) } \\
\text { Estudios primarios o básicos } \\
\text { Secundarios } \\
\text { Título universitario o superior } \\
\end{array}$ & $\begin{array}{l}25,6 \\
46,2 \\
28,1 \\
\end{array}$ & $\begin{array}{l}14,3 \\
48,6 \\
37,1 \\
\end{array}$ & $\begin{array}{l}28,1 \\
45,7 \\
26,2 \\
\end{array}$ & $\begin{array}{c}\chi^{2}=5,07 \\
g l=9 \\
p<, 828\end{array}$ \\
\hline
\end{tabular}

Tabla B. Características clínicas del Grupo de adicción (GA) y el Grupo general de psiquiatría (GGP).

\begin{tabular}{|c|c|c|c|}
\hline \multicolumn{2}{|l|}{ Grupo de adicción $n=36$} & \multicolumn{2}{|l|}{ Grupo general de psiquiatría $\mathrm{n}=166$} \\
\hline Trastorno por uso de alcohol $n=30$ & $83,3 \%$ & Esquizofrenia y otros trastornos psiquiátricos $n=79$ & $47,6 \%$ \\
\hline Trastorno por uso de sedantes $n=14$ & $38,9 \%$ & Trastorno del estado de ánimo (trastorno depresivo o bipolar) $n=61$ & $36,7 \%$ \\
\hline Trastorno por uso de cocaína $\mathrm{n}=10$ & $27,8 \%$ & Trastorno inducido por uso de sustancias $n=6$ & $3,6 \%$ \\
\hline Trastorno por uso de cannabis $n=6$ & $16,7 \%$ & Miscelánea $\mathrm{n}=20$ & $12,0 \%$ \\
\hline Trastorno por uso de opioides $n=6$ & $16,7 \%$ & Patología dual $\mathrm{n}=38$ & $22,9 \%$ \\
\hline Trastorno por uso de nicotina $n=24$ & $66,7 \%$ & & \\
\hline Patología dual $n=17$ & $47,2 \%$ & & \\
\hline Policonsumo de drogas $n=20$ & $55,6 \%$ & & \\
\hline
\end{tabular}

Nota. Diagnóstico según criterios del DSM-IV. El grupo de adicciones (GA) incluye a pacientes ingresados para la desintoxicación de alcohol u otras sustancias. El grupo de psiquiatría general (GGP) incluye a los pacientes ingresados en la misma unidad de hospitalización psiquiátrica debido a un trastorno mental diferente de TUS.

Tabla C. Coeficientes $\alpha$ de Cronbach y Omega de McDonald

\begin{tabular}{lcr}
\hline & $\alpha$ de Cronbach & Omega de McDonald \\
\hline Puntuación total de riesgo &, 92 &, 93 \\
TSI sustancias ilegales &, 91 &, 93 \\
SSI tabaco &, 88 &, 89 \\
SSI alcohol &, 93 &, 92 \\
SSI cannabis &, 95 &, 95 \\
SSI cocaína &, 96 &, 93 \\
SSI anfetaminas &, 93 &, 89 \\
SSI sedantes &, 95 &, 96 \\
SSI opioides &, 96 &, 94 \\
\hline
\end{tabular}


alcohol y anfetaminas fueron congruentes con los obtenidos en el estudio con pacientes con un primer episodio psicótico (Hides et al., 2009), mientras que el punto de corte para alcohol en el estudio con población adolescente (Gryczynski et al., 2015) fue menor.

En este estudio, el punto de corte óptimo para cannabis es más alto que el obtenido en los estudios antes mencionados. Esto puede deberse a la presencia de solo dos sujetos con diagnóstico de dependencia en el GA en comparación con la alta proporción de sujetos con trastorno por consumo de cannabis en el GPG.

En comparación con el estudio de validación original (Humeniuk et al., 2008), los puntos de corte propuestos obtuvieron puntuaciones de sensibilidad y especificidad superiores o similares. Al utilizar las puntuaciones de corte de la OMS, los valores se mantuvieron similares a los de estudios anteriores, excepto por los puntos de corte de alto riesgo para cannabis y sedantes, que eran más bajos.

Para muchas sustancias, como cannabis, la validación en esta población sensible es una clara necesidad (López-Pelayo, Batalla, Balcells, Colom y Gual, 2015). Entre las ventajas del ASSIST, podemos destacar su menor tiempo de aplicación en comparación con MINI (Ferrando et al., 1998) o PRISM (Torrens, Serrano, Astals, Pérez-Domínguez y Martín-Santos, 2004), y que, a diferencia de otras pruebas adaptadas a la población psiquiátrica, abarca todas las sustancias. Por ejemplo, el DALI (Rosenberg et al., 1998) no criba para tabaco, anfetaminas, sedantes u opioides, y el DAST (Pérez-Gálvez et al., 2010) no incluye alcohol ni tabaco. El hecho de que este estudio se haya realizado con pacientes que padecen un episodio agudo, cuyos procesos cognitivos y capacidad para completar un test podrían verse comprometidos, muestra que puede aplicarse en entornos hospitalarios y en pacientes que padecen no solo un primer episodio psicótico sino también otras enfermedades mentales. El cribado de TUS con instrumentos validados en pacientes con trastorno psiquiátrico (Greenberg y Rosenheck, 2014; Langås, Malt y Opjordsmoen, 2011b, 2011a; Torrens, Martin-Santos y Samet, 2006) y otras poblaciones vulnerables que pueden tener una patología dual (Vargas-Cáceres et al., 2020) es importante para identificar con precisión conductas de riesgo relacionadas con el uso de sustancias, diagnosticar y realizar una intervención breve motivacional o derivar a tratamiento especializado. Una intervención temprana puede mejorar el curso de la enfermedad.

El presente estudio tiene varias limitaciones, siendo la más relevante el tamaño muestral y la resultante pequeña representación de determinadas sustancias como inhalantes, alucinógenos o anfetaminas que imposibilitaban el cálculo de sensibilidad y especificidad para algunas sustancias. El reclutamiento fue únicamente en un entorno hospitalario, por lo que la generalización de los resultados es modesta. Sin embargo, ambas limitaciones son comunes en los estudios de validación. La fiabilidad test-retest no se realizó por- que los pacientes fueron dados de alta poco después de la primera administración. Tampoco se calcularon evidencias de validez predictiva. Una limitación adicional es que el uso de cuestionarios similares a los de estudios anteriores para evaluar las correlaciones con las puntuaciones del ASSIST introduce un posible sesgo de redundancia. Por último, la muestra se limitó a pacientes hospitalizados. La generalización a pacientes con problemas de salud mental no debería ser difícil debido a sus síntomas más leves y su mejor estado cognitivo. No se han recopilado evidencias de validez para la versión autoadministrada del ASSIST.

A pesar de estas limitaciones, las excelentes propiedades de la versión española del ASSIST en población diagnosticada de un trastorno psiquiátrico favorecen su aplicación como parte de la práctica habitual. El estudio tiene varias fortalezas. Primero, abarca un amplio rango de trastornos mentales y de sustancias. Segundo, se utilizó un método de validación bien establecido (Humeniuk et al., 2008). La inclusión de todos los parámetros relevantes de validación en un mismo estudio no es común (López-Pelayo et al., 2015). Teniendo en cuenta que un riesgo moderado en una población de atención primaria puede considerarse como un alto riesgo en pacientes con trastorno psiquiátrico, es importante la detección del uso problemático (riesgo moderado) e intervención temprana en esta población.

La versión en español de ASSIST está disponible para mejorar la detección e intervención temprana en los trastornos por uso de sustancias en pacientes con trastorno psiquiátrico hospitalizados. Su aplicación puede ayudar a reducir reingresos y recaídas, aumentar la adherencia al tratamiento y mejorar la calidad de vida.

El ASSIST mostró una buena evidencia de validez y fiabilidad para evaluar el nivel de riesgo de uso de sustancias en pacientes psiquiátricos hospitalizados.

\section{Reconocimientos}

Los autores agradecen a los profesionales sanitarios de la Unidad de Psiquiatría del Hospital Clínic de Barcelona que estaban tratando a los participantes del estudio y colaboraron en aportar información clínica y en la recogida de datos.

Este trabajo ha sido financiado por el Programa CERCA/Generalitat de Catalunya. H. López-Pelayo recibió financiación del Ministerio de Ciencia, Innovación y Universidades de España, Instituto de Salud Carlos III a través de un contrato Juan Rodes (JR19/00025).

\section{Conflicto de intereses}

H. López-Pelayo ha recibido honorarios de Lundbeck, Teva y Janssen y becas de viaje de Otsuka, Lundbeck y Exeltis. Ninguno de estos apoyos representa conflicto de interés alguno con la información proporcionada en este estudio. 
Los demás autores declaran la inexistencia de conflicto de interés.

\section{Referencias}

Ali, R., Awwad, E., Babor, T. F., Bradley, F., Butau, T., Farrell, M.,... Vendetti, J. (2002). The Alcohol, Smoking and Substance Involvement Screening Test (ASSIST): Development, reliability and feasibility. Addiction, 97, 1183-1194. doi:10.1046/j.1360-0443.2002.00185.x.

American Educational Research Association, American Psychological Association, y National Council on Measurement in Education. (2014). Standards for Educational and Psychological Testing. Washington, DC: American Educational Research Association.

Bascarán, M. T. B., Flórez, G., Seijo, P. y García, J. B. (2019). Does icd-11 improve the epidemiological and nosological purposes of mental, behavioral and developmental disorders? Adicciones, 31, 183-188. doi:10.20882/adicciones.1368,

Becoña, E. y Vázquez, F. L. (1998). The Fagerström Test for Nicotine Dependence in a Spanish sample. Psychological Reports, 83, 1455-1458. doi:10.2466/pr0.1998.83.3f.1455.

Cohen, J. (1988). Statistical Power Analysis for the Behavioral Sciences. New York: Routledge Academic.

Conway, K. P., Compton, W., Stinson, F. S. y Grant, B. F. (2006). Lifetime comorbidity of DSM-IV mood and anxiety disorders and specific drug use disorders: Results from the National Epidemiologic Survey on Alcohol and Related Conditions. Journal of Clinical Psychiatry, 67, 247257. doi:10.4088/jcp.v67n0211.

Crome, I. B., Bloor, R. y Thom, B. (2006). Screening for illicit drug use in psychiatric hospitals: Whose job is it? Advances in Psychiatric Treatment, 12, 375-383. doi:10.1192/ apt.12.5.375.

Cronbach, L. J. (1951). Coefficient alpha and the internal structure of tests. Psychometrika, 16, 297-334. doi:10.1007/ BF02310555.

Degenhardt, L., Bharat, C., Glantz, M. D., Sampson, N. A., Scott, K., Lim, C. C. W.,... Kessler, R. C. (2019). The epidemiology of drug use disorders cross-nationally: Findings from the WHO's World Mental Health Surveys. International Journal of Drug Policy, 71, 103-112. doi:10.1016/j. drugpo.2019.03.002.

Degenhardt, L., Glantz, M., Evans-Lacko, S., Sadikova, E., Sampson, N., Thornicroft, G.,... Zaslavsky, A. M. (2017). Estimating treatment coverage for people with substance use disorders: An analysis of data from the World Mental Health Surveys. World Psychiatry, 16, 299-307. doi:10.1002/wps.20457.

Díaz-Mesa, E., Portilla, P. G., Sáiz, P. A., Bascarán, T. B., Casares, M. J., Fonseca, E.,... Bobes, J. (2010). Psychometric performance of the 6th version of the Addiction Severity Index in Spanish (ASI-6). Psicothema, 22, 513-519.
Dixon, L. (1999). Dual diagnosis of substance abuse in schizophrenia: Prevalence and impact on outcomes. Schizophrenia Research, 35, 93-100. doi:10.1016/s09209964(98)00161-3.

Dunn, T. J., Baguley, T. y Brunsden, V. (2014). From alpha to omega: A practical solution to the pervasive problem of internal consistency estimation. British Journal of Psychology, 105, 399-412. doi:10.1111/bjop.12046.

Ferrando, L., Franco, A., Soto, M., Bobes, J., Soto, O., Franco, L. y Gubert, J. (1998). MINI International Neuropsychiatric Interview (Spanish version 5.0.0.) DSM-IV. Madrid: Instituto IAP.

González-Sáiz, F. y Carulla-Salvador, L. (1998). Estudio de fiabilidad y validez de la versión española de la escala Severity of Dependence Scale (SDS). Adicciones, 10, 223232.

Greenberg, G. A. y Rosenheck, R. A. (2014). Psychiatric correlates of past incarceration in the national co-morbidity study replication. Criminal Behaviour and Mental Health, 24, 18-35. doi:10.1002/cbm.1875.

Gryczynski, J., Kelly, S. M., Mitchell, S. G., Kirk, A., O’Grady, K. E. y Schwartz, R. P. (2015). Validation and performance of the Alcohol, Smoking and Substance Involvement Screening Test (ASSIST) among adolescent primary care patients. Addiction, 110, 240-247. doi:10.1111/add.12767.

Harris, M. G., Bharat, C., Glantz, M. D., Sampson, N. A., Al-Hamzawi, A., Alonso, J.,... Degenhardt, L. (2019). Cross-national patterns of substance use disorder treatment and associations with mental disorder comorbidity in the WHO World Mental Health Surveys. Addiction, 114, 1446-1459. doi:10.1111/add.14599.

Hides, L., Cotton, S. M., Berger, G., Gleeson, J., O'donnell, C., Proffitt, T.,... Lubman, D. I. (2009). The reliability and validity of the Alcohol, Smoking and Substance Involvement Screening Test (ASSIST) in first-episode psychosis. Addictive Behaviors, 34, 821-825. doi:10.1016/j. addbeh.2009.03.001.

Hu, L. T. y Bentler, P. M. (1999). Cutoff criteria for fit indexes in covariance structure analysis: Conventional criteria versus new alternatives. Structural Equation Modeling, 6, 1-55. doi:10.1080/10705519909540118.

Humeniuk, R., Ali, R., Babor, T. F., Farrell, M., Formigoni, M. L., Jittiwutikarn, J.,... Simon, S. (2008). Validation of the alcohol, smoking and substance involvement screening test (ASSIST). Addiction, 103, 1039-1047. doi:10.1111/ j.1360-0443.2007.02114.x.

IBM Corp. Released. (2019). IBM SPSS Statistics for Windows, Version 26.0. Armonk, NY: IBM Corp.

Langås, A. M., Malt, U. F. y Opjordsmoen, S. (2011a). Comorbid mental disorders in substance users from a single catchment area - a clinical study. BMC Psychiatry, 11, 25. doi:10.1186/1471-244X-11-25.

Langås, A. M., Malt, U. F. y Opjordsmoen, S. (2011b). Substance use disorders and comorbid mental disorders in 
first-time admitted patients from a catchment area. European Addiction Research, 18, 16-25. doi:10.1159/000332234.

Lobo, A., Ezquerra, J., Gómez, F., Sala, J. y Seva, A. (1979). El Mini Examen Cognoscitivo. Un test sencillo y práctico para detectar alteraciones intelectuales en pacientes médicos. Actas Luso-Españolas de Neurología, Psiquiatría y Ciencias Afines, 3, 189-202.

López-Pelayo, H., Batalla, A., Balcells, M. M., Colom, J. y Gual, A. (2015). Assessment of cannabis use disorders: A systematic review of screening and diagnostic instruments. Psychological Medicine, 45, 1121-1133. doi:10.1017/ S0033291714002463.

Merikangas, K. R., Jin, R., He, J. P., Kessler, R. C., Lee, S., Sampson, N. A.,... Zarkov, Z. (2011). Prevalence and correlates of bipolar spectrum disorder in the World Mental Health Survey Initiative. Archives of General Psychiatry, 68, 241-251. doi:10.1001/archgenpsychiatry.2011.12.

Morisano, D., Babor, T. F. y Robaina, K. A. (2014). Co-occurrence of substance use disorders with other psychiatric disorders: Implications for treatment services. Nordic Studies on Alcohol and Drugs, 31, 5-25. doi:10.2478/nsad2014-0002.

Muñiz, J. y Fonseca-Pedrero, E. (2019). Ten steps for test development. Psicothema, 31, 7-16. doi:10.7334/psicothema2018.291.

Nunnally, J. C. y Bernstein, I. H. (1994). Psychometric theory. New York: McGraw-Hill.

Pérez-Gálvez, B., García-Fernández, L., de Vicente-Manzanaro, M. P., Oliveras-Valenzuela, M. A. y Lahoz-Lafuente, M. (2010). Spanish validation of the Drug Abuse Screening Test (DAST-20 y DAST-10). Health and Addictions, 10, 3550. doi:10.21134/haaj.v10i1.35.

Pérez-Moreno, P., Calzada-Álvarez, N., Rovira-Guardiola, J. y Torrico Linares, E. (2012). Estructura factorial del test ASSIST: Aplicación del análisis factorial exploratorio y confirmatorio. Trastornos Adictivos, 14, 44-49. doi:10.1016/ S1575-0973(12)70043-0.

R Core Team. (2020). R: A Language and Environment for Statistical Computing, version 3.5. Vienna, Austria.

Rosenberg, S. D., Drake, R. E., Wolford, G. L., Mueser, K. T., Oxman, T. E., Vidaver, R. M.,... Luckoor, R. (1998). Dartmouth Assessment of Lifestyle Instrument (DALI): A substance use disorder screen for people with severe mental illness. American Journal of Psychiatry, 155, 232-238. doi:10.1176/ajp.155.2.232.

Rubio Valladolid, G., Bermejo Vicedo, J., Caballero Sánchez-Serrano, M. C. y Santo-Domingo Carrasco, J. (1998).
Validación de la prueba para la identificación de trastornos por uso de alcohol (AUDIT) en Atención Primaria. Revista Clinica Española, 198, 11-14.

Rubio Valladolid, G., Martínez-Raga, J., Martínez-Gras, I., Ponce Alfaro, G., de la Cruz Bértolo, J., Jurado Barba, R.,... Zarco Montejo, J. (2014). Validación de la versión española del Test de Detección de Uso de Alcohol, Tabaco y otras Sustancias (ASSIST). Psicothema, 26, 180-185. doi:10.7334/psicothema2013.172.

Sheehan, D. V., Lecrubier, Y., Sheehan, K. H., Amorim, P., Janavs, J., Weiller, E.,... Dunbar, G. C. (1998). The Mini-International Neuropsychiatric Interview (M.I.N.I.): The development and validation of a structured diagnostic psychiatric interview for DSM-IV and ICD-10. Journal of Clinical Psychiatry, 59, 22-33.

Tiburcio Sainz, M., Rosete-Mohedano, M. G., Natera Rey, G., Martínez Vélez, N. A., Carreño García, S. y Pérez Cisneros, D. (2016). Validity and reliability of the alcohol, smoking, and substance involvement screening test (ASSIST) in university students. Adicciones, 28, 19-27. doi:10.20882/ adicciones. 786.

Torrens, M., Martin-Santos, R. y Samet, S. (2006). Importance of clinical diagnoses for comorbidity studies in substance use disorders. Neurotoxicity Research, 10, 253-261. doi:10.1007/BF03033361.

Torrens, M., Serrano, D., Astals, M., Pérez-Domínguez, G. y Martín-Santos, R. (2004). Diagnosing comorbid psychiatric disorders in substance abusers: Validity of the Spanish versions of the psychiatric research interview for substance and mental disorders and the structured clinical interview for DSM-IV. American Journal of Psychiatry, 161, 1231-1237. doi:10.1176/appi.ajp.161.7.1231.

Vargas-Cáceres, S., Mantilla, M. F., Ortega, G., Bruguera, E., Casas, M., Ramos-Quiroga, J.-A. y Braquehais, M. D. (2020). Diagnóstico dual en médicos residentes: Una revisión sistemática. Adicciones, 32, 281-290. doi:10.20882/ adicciones. 1253 .

Vélez-Moreno, A., González-Saiz, F., Rojas, A. J., Torrico-Linares, E., Fernández-Calderón, F., Ramírez-López, J. y Lozano, Ó. M. (2015). Reliability and validity of the Spanish version of the substance dependence severity scale. European Addiction Research, 21,39-46. doi:10.1159/000365282.

World Medical Association Declaration of Helsinki. (2013). World Medical Association declaration of Helsinki: Ethical principles for medical research involving human subjects. Journal of the American Medical Association, 310, 21912194. doi:10.1001/jama.2013.281053. 
